\title{
The Baikal Neutrino Telescope: From NT200 to NT200+
}

\author{
V.Aynutdinov ${ }^{a}$, V. BAlkAnov ${ }^{a}$, I. Belolaptikov ${ }^{g}$, L. Bezrukov $^{a}$,

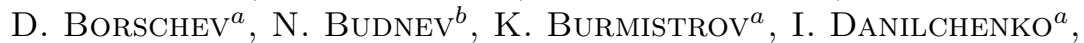 \\ Ya. Davidov $^{a}$, G. Domogatsky ${ }^{a}$, A. Doroshenko $^{a}$, A. DyachoK $^{b}$, \\ Zh.-A. Dzhilkibaev ${ }^{a}$, S. Fialkovsky ${ }^{d}$, O. Gaponenko ${ }^{a}, \mathrm{~K}$ Golubkov $^{a}$,

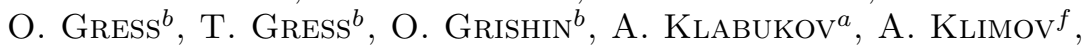 \\ A. Kochanov ${ }^{b}, \mathrm{~K} . \mathrm{KonischeV}^{g}$, A. KoshechKin ${ }^{a}, \mathrm{~V} \cdot \mathrm{KulePoV}^{d}$, \\ L. Kuzmichev ${ }^{c}, \mathrm{Vy}_{\text {y }} \mathrm{Kuznetzov}^{a}$, B. Lubsandorzhiev ${ }^{a}$, S. Mikheyev ${ }^{a}$, \\ M. Milenin ${ }^{d}$, R. Mirgazov ${ }^{b}$, T. Mikolajsiki ${ }^{h}$, E. Osipova ${ }^{c}$, A. Panfilov $^{a}$,

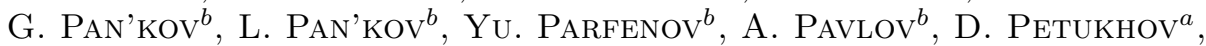 \\ E. Pliskovsky ${ }^{g}, \mathrm{P} . \mathrm{Pokhil}^{a}$, V. Poleschuk ${ }^{a}, \mathrm{E} \cdot \mathrm{Popova}^{c}, \mathrm{~V} \cdot \mathrm{Prosin}^{c}$, \\ M. Rosanov ${ }^{e}$, V. Rubtzov ${ }^{b}$, B. Shaibonov ${ }^{a}$, A. Shirokov ${ }^{c}$, Ch. Spiering $^{h}$,

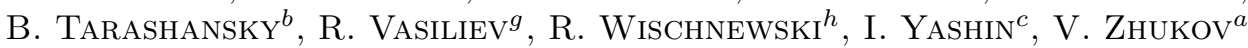 \\ ${ }^{a}$ Institute for Nuclear Research, Russian Academy of Sciences, Moscow, Russia \\ ${ }^{b}$ Irkutsk State University, Irkutsk, Russia \\ ${ }^{c}$ Skobeltsyn Institute of Nuclear Physics MSU, Moscow, Russia \\ ${ }^{d}$ Nizhni Novgorod State Technical University, Nizhni Novgorod, Russia \\ e St.Peterburg State Marine Technical University, St.Petersburg, Russia \\ ${ }^{f}$ Kurchatov Institute, Moscow, Russia \\ ${ }^{g}$ Joint Institute for Nuclear Research, Dubna, Russia \\ ${ }^{h}$ DESY, Zeuthen, Germany
}

Received Nov 30, 2005

We review the status of the Baikal Neutrino Telescope, which has recently been upgraded to the 5 Mton detector NT200+. We present results on searches for upward going atmospheric neutrinos and relativistic magnetic monopoles, obtained from 1998-2002 with the predecessor detector NT200. A search for very high energy neutrinos yields an upper limit on the extraterrestrial diffuse neutrino flux for $20 \mathrm{TeV}<\mathrm{E}<50 \mathrm{PeV}$. We describe the strategy of upgrading NT200 to NT200+ and creating a detector on the Gigaton (km3) scale at lake Baikal. R\&D activities on that next stage detector have been started.

PACS: 95.85.Ry, 14.80.Hv

Key words: neutrino telescope, high energy neutrinos, magnetic monopoles

\section{Introduction}

The Baikal Neutrino Telescope is operated in Lake Baikal, Siberia, at a depth of $1.1 \mathrm{~km}$. The first stage telescope configuration NT200 [1] was put into permanent operation on April 6th, 1998 and consists of 192 optical modules (OMs). An umbrella-like frame carries 8 strings, each with 24 pairwise arranged OMs (see central part of Fig. 1). Four underwater electrical cables connect the detector with the shore station. Each optical module contains a 37-cm diameter QUASAR - photomultiplier (PM) which has been developed specially for this project [2]. The two $\mathrm{PMs}$ of a pair are switched in local coincidence in order to suppress background 


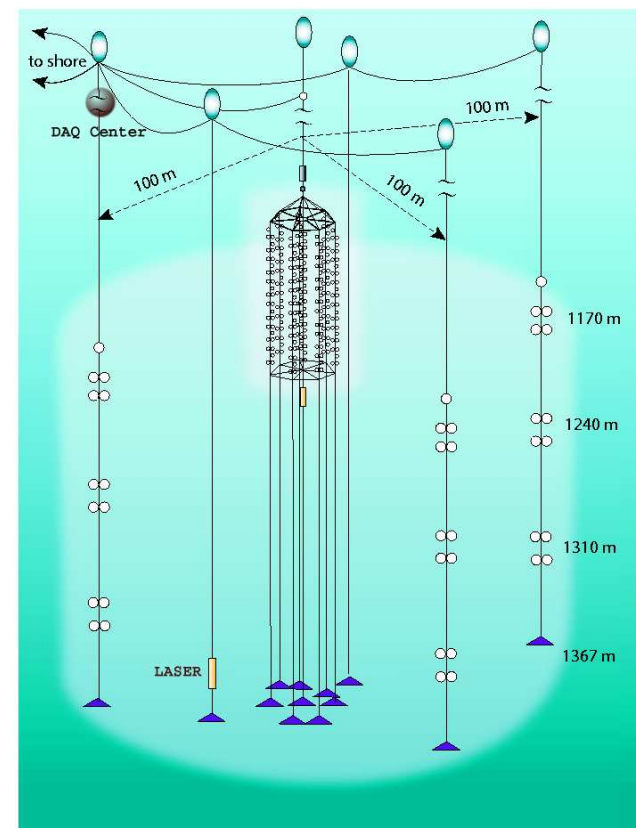

Fig. 1. The upgraded Baikal Telescope NT200+ : The old NT200 surrounded by three external long strings at $100 \mathrm{~m}$ radius from the center. Also indicated: external laser and DAQ center.

from bioluminescence and PM noise; each pair defines a channel.

The upgraded telescope NT200+ was put into operation on April 9th, 2005. This configuration consists of the old NT200 telescope, surrounded by three new, external strings placed $100 \mathrm{~m}$ from the center of NT200 (see Fig.1). With these new strings, the sensitivity of Baikal telescope for very high energy neutrinos increases by a factor 4 .

We are presenting new results obtained with NT200 including data taken from 1998-2002: atmospheric muon neutrino studies and searches for relativistic magnetic monopoles and extraterrestrial high energy neutrinos. We also describe the data acquisition, control and calibration systems of the new NT200+ telescope. NT200+ will be used as the the basic cell of a future Gigaton detector in Lake Baikal, which is briefly sketched.

\section{Atmopsheric Muon Neutrinos}

The signature of charged current muon neutrino events is a muon crossing the detector from below. Muon track reconstruction algorithms and background rejection have been described elsewhere [3]. Compared to [3], the analysis of the 5-year 
sample (1038 days of live time from April, 1998 - February, 2003) was optimized for higher signal passing rate, and accepting a slightly larger contamination of $15-20 \%$ fake events. A total of 372 upgoing neutrino candidates were found. From MonteCarlo simulations, 385 atmospheric neutrino and background events are expected. The skyplot of these events is shown in Fig. 2.

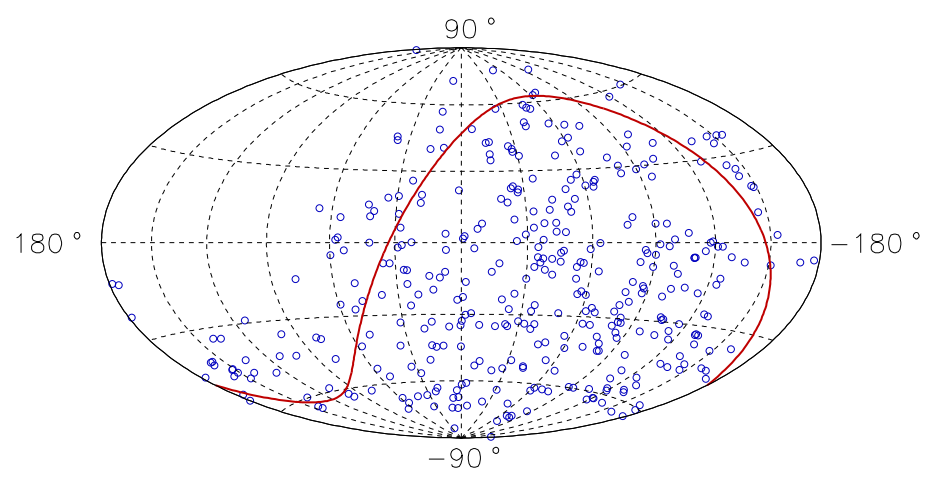

Fig. 2. Skyplot (galactic coordinates) of neutrino events for five years. The solid curve shows the equator.

\section{Search for Relativistic Magnetic Monopoles}

Fast magnetic monopoles with Dirac charge $g=68.5 e$ are interesting objects to search for with deep underwater neutrino telescopes. The intensity of monopole Cherenkov radiation is $\approx 8300$ times higher than that of muons. Optical modules of the Baikal experiment can detect such an object from a distance up to hundred meters. The processing chain for fast monopoles starts with the selection of events with a high multiplicity of hit channels: $N_{\text {hit }}>30$. In order to reduce the background from downward atmospheric muons we restrict ourself to monopoles coming from the lower hemisphere. For an upward going particle the times of hit channels increase with rising z-coordinates from bottom to top of the detector. To suppress downward moving particles, a cut on the value of the time- $\mathrm{Z}^{-}$-correlation, $\operatorname{cor}_{T z}$, is applied:

$$
\operatorname{cor}_{T z}=\frac{\sum_{i=1}^{N_{h i t}}\left(t_{i}-\bar{T}\right)\left(z_{i}-\bar{z}\right)}{N_{h i t} \sigma_{t} \sigma_{z}}>0
$$

where $t_{i}$ and $z_{i}$ are time and z-coordinate of a fired channel, $\bar{T}$ and $\bar{z}$ are mean values for times and z-coordinates of the event and $\sigma_{t}$ and $\sigma_{z}$ the rms-errors for time and z-coordinates. 


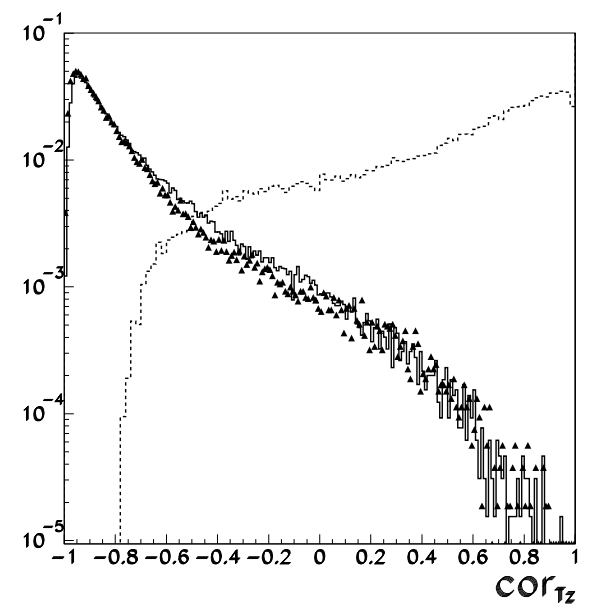

Fig. 3. $\operatorname{cor}_{T z}$ distributions for experimental events (triangles), simulated atmospheric muon evens (solid), and simulated upward moving relativistic magnetic monopoles (dotted); multiplicity cut

$$
N_{\text {hit }}>30 \text {. }
$$

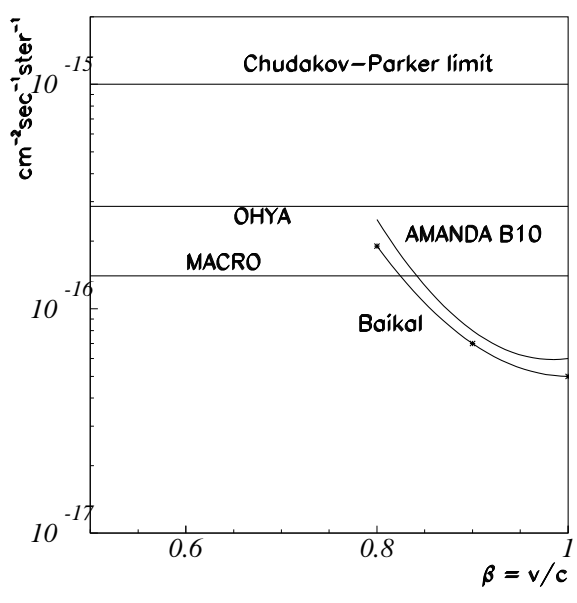

Fig. 4. Upper limits on the flux of relativistic magnetic monopoles as obtained in this analysis and in other experiments.

In Fig. 3 we compare the $\operatorname{cor}_{T z}$-distribution for experimental data (triangles), simulated atmospheric muon events (solid curve) with simulated upward moving monopole events (dotted curve).

Within 994 days of live time, about $3 \cdot 10^{8}$ events with $N_{\text {hit }}>4$ have been recorded, with 20943 of them satisfying cut $0\left(N_{h i t}>30\right.$ and $\left.\operatorname{cor}_{T z}>0\right)$. For further background suppression (see [23] for details of the analysis) we use additional cuts, which essentially reject muon events and at the same time only slightly reduce the effective area for relativistic monopoles:

1. $N_{\text {hit }}>35$ and $\operatorname{cor}_{T z}>0.4 \div 0.6$

2. $\chi^{2}$ determined from reconstruction has to be smaller than 3

3. Reconstructed zenith angle $\theta>100 \mathrm{deg}$

4. Reconstructed track distance from NT200 center $R_{r e c}>20 \div 25 \mathrm{~m}$.

No events from the experimental sample pass cuts 1-4.

The acceptances $A_{\text {eff }}$ for monopoles with $\beta=1,0.9,0.8$ have been calculated for all NT200 operation configurations (various sets of operating channels, with cuts optimized for each configuration). For the time periods included, $A_{\text {eff }}$ varies between $3 \cdot 10^{8}$ and $6 \cdot 10^{8} \mathrm{~cm}^{2} \operatorname{sr}($ for $\beta=1$ ).

From the non-observation of candidate events in NT200 and the earlier stage telescopes NT36 and NT96 [4], a combined upper limit on the flux of fast monopoles 
Table 1. $A_{e f f} * T$ and $90 \%$ C.L. upper limits on the flux of fast monopoles.

\begin{tabular}{||c|c|c|c||}
\hline \hline & $\beta=1$ & $\beta=0.9$ & $\beta=0.8$ \\
\hline $\begin{array}{c}\mathrm{NT} 200 A_{\text {eff }} \cdot T \\
{\left[\mathrm{~cm}^{2} \cdot \mathrm{sec} \cdot \mathrm{sr}\right]}\end{array}$ & $4.53 \cdot 10^{16}$ & $3.22 \cdot 10^{16}$ & $1.18 \cdot 10^{16}$ \\
\hline $\begin{array}{c}\mathrm{NT} 36+\mathrm{NT} 96 A_{\text {eff }} \cdot T \\
{\left[\mathrm{~cm}^{2} \cdot \mathrm{sec} \cdot \mathrm{sr}\right]}\end{array}$ & $0.37 \cdot 10^{16}$ & $0.25 \cdot 10^{16}$ & $0.9 \cdot 10^{15}$ \\
\hline $\begin{array}{c}90 \% \text { C.L. upper flux limit } \\
{\left[\mathrm{cm}^{-2} \cdot \mathrm{sec}^{-1} \cdot \mathrm{sr}^{-1}\right]}\end{array}$ & $0.5 \cdot 10^{-16}$ & $0.7 \cdot 10^{-16}$ & $1.92 \cdot 10^{-16}$ \\
\hline
\end{tabular}

with $90 \%$ confidence level is obtained. The cumulative acceptances $A_{\text {eff }} \cdot T$, as well as the $90 \%$ C.L. upper flux limits are presented in Table 1.

In Fig. 4 we compare this 90\% C.L. upper limit for an isotropic flux of fast monopoles obtained with the Baikal neutrino telescope to the final limits from the underground experiments Ohya [5] and MACRO [6] and to the limit reported for the underice detector AMANDA[7]. The Baikal limit is currently the most stringent one.

\section{Search for Extraterrestrial Neutrinos}

We present results of a search for diffuse neutrinos with energies larger than 10 $\mathrm{TeV}$. The analysis is based on data taken with the Baikal neutrino telescope NT200 in the five years 1998-2002. Instead of focusing on high energy muons crossing the array, this analysis is tailored to signatures of isolated high-energy cascades in a large volume around the detector (for a detailed description see [22]). Within the 1038 days of detector live time between April 1998 and February 2003, $3.45 \times 10^{8}$ events with $N_{\text {hit }} \geq 4$ have been recorded. For this analysis we used 22597 events with hit channel multiplicity $N_{\text {hit }}>15$ which obey the condition:

$$
t_{\min }=\min \left(t_{i}-t_{j}\right)>-10 \mathrm{~ns}, \quad i<j .
$$

Here, $t_{i}, t_{j}$ are the light arrival times at channels $i, j$ (the numbering of channels is from top to bottom along the string). The distribution of experimental events is consistent with the background simulation. No statistically significant excess above the background from atmospheric muons has been observed. With no experimental events outside the area populated by background events in the $\left(t_{\mathrm{min}}, N_{\text {hit }}\right)$ parameter space we derive upper limits on the fluxes of high energy neutrinos as predicted by different models of neutrino sources. For an $E^{-2}$ behaviour of the neutrino spectrum and a flavor ratio $\nu_{e}: \nu_{\mu}: \nu_{\tau}=1: 1: 1$ (at Earth, after oscillation), the $90 \%$ C.L. upper limit on the neutrino flux of all flavors obtained with the Baikal 
neutrino telescope NT200 (1038 days) is:

$$
E^{2} \Phi<8.1 \times 10^{-7} \mathrm{~cm}^{-2} \mathrm{~s}^{-1} \mathrm{sr}^{-1} \mathrm{GeV}
$$

Assuming an upper limit on the number of signal events $n_{90 \%}=2.5$, the modelindependent limit on $\overline{\nu_{e}}$ at the $\mathrm{W}$ - resonance energy is:

$$
\Phi_{\overline{\nu_{e}}}<3.3 \times 10^{-20} \mathrm{~cm}^{-2} \mathrm{~s}^{-1} \mathrm{sr}^{-1} \mathrm{GeV}^{-1} .
$$

Figure 5 shows our upper limit on a $\left(\nu_{e}+\nu_{\mu}+\nu_{\tau}\right) E^{-2}$ diffuse flux as well as the model independent limit on the resonant $\bar{\nu}_{e}$ flux (diamond). Also shown are the limits obtained by MACRO and by cascade-like searches in AMANDA [8, 9, 10, 11], theoretical bounds obtained by Berezinsky (model independent (B) [12] and for an $E^{-2}$ shape of the neutrino spectrum $\left(\mathrm{B}\left(E^{-2}\right)\right)[13]$, by Waxman and Bahcall (WB) [14], by Mannheim et al. (MPR) [15], predictions for neutrino fluxes from topological defects (TD) [17], a prediction for a diffuse flux from AGNs according to Nellen et al. (NMB) [18], as well as the atmospheric conventional neutrino fluxes [19] from horizontal and vertical directions (atm; upper and lower curves, respectively) and the atmospheric prompt neutrino flux obtained by Volkova et al. $\left(\nu_{p r}\right)$ [20]. Our upper limits (solid curves) on diffuse fluxes from AGNs shaped according to the model of Stecker and Salamon (SS) [16] and of Semikoz and Sigl (SeSi) [17] are shown in Fig. 6.

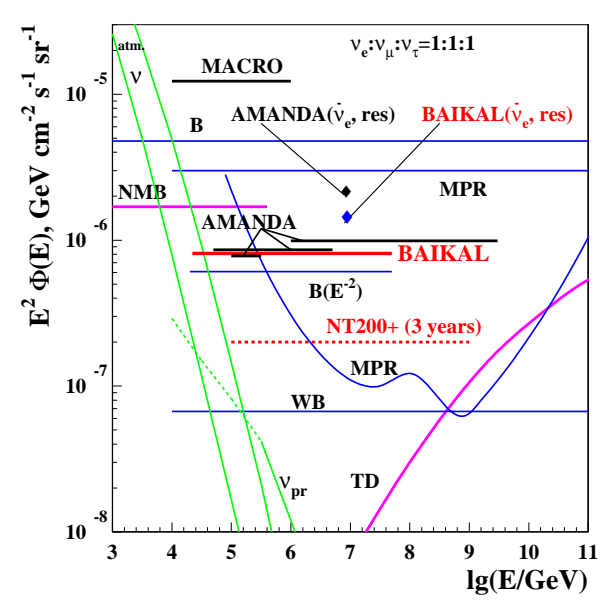

Fig. 5. Neutrino flux predictions in different models of neutrino sources compared to experimental upper limits to $E^{-2}$ fluxes obtained by various experiments.

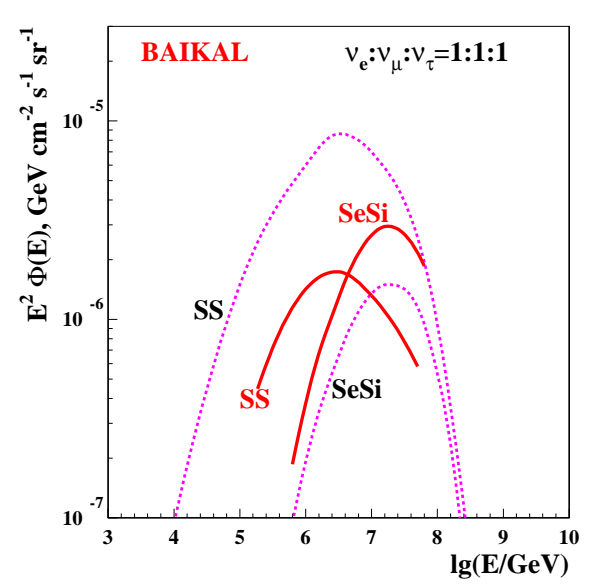

Fig. 6. Experimental limits compared to two model predictions. Dotted curves: predictions from model SS [16] and SeSi [17]. Full curves: upper limits to spectra of the same shape. Model SS is excluded, model SeSi is not. 


\section{NT200+ Data Acquisition and Control Systems}

The upgraded telescope NT200+ was put into operation on April 9th, 2005. This new configuration consists of a central part (the old telescope NT200) and three new, external strings (NT+), see Fig. 1. The external strings are $140 \mathrm{~m}$ long and are placed at 100 meter distance from the center of NT200. Each string contains 12 OMs grouped in pairs like in NT200. The upper pairs are at approximately the same level as the bottom OMs of NT200.

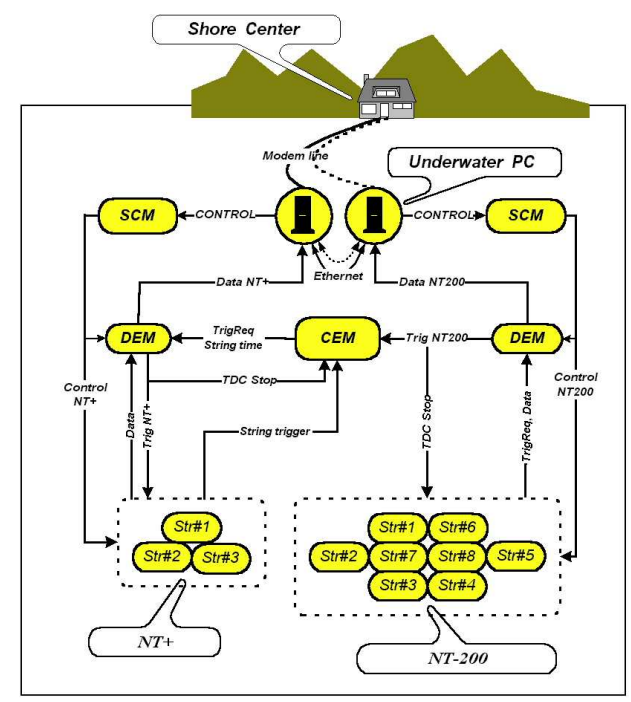

Fig. 7. Sketch of data collection and slow control in NT200+: 8-string telescope NT200 and 3 new outer strings (NT+), controlled from two underwater PCs.

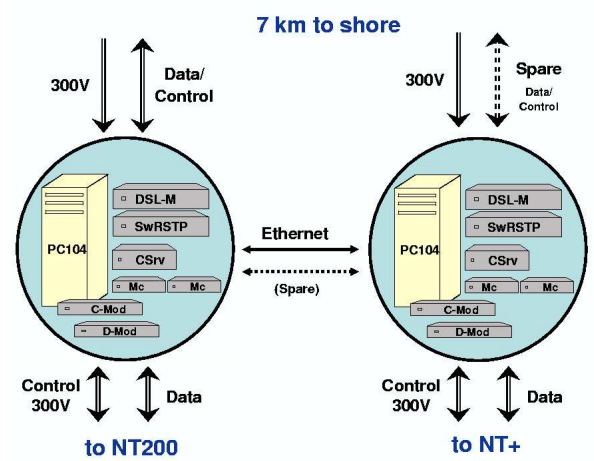

Fig. 8. Sketch of the new NT200+ central DAQ/Control spheres with embedded $\mathrm{PC} 104$.

It was necessary to solve two basic tasks for integrating NT200 and the external string subsystems into a united detector: creation of a data acquisition and control system for the external strings, and providing a time synchronisation between the two subsystems and a tagging of common events (see also [24]). Since a simple doubling of the data acquisition and control system for NT200 and NT+ was compatible neither with the number of available cable connections to shore, nor with future upgrades, we decided to significantly modernize the system by introducing for the first time embedded PCs with reliable industrial Ethernet infrastructure underwater. For NT200+, all data and control cable connections of NT200 and the outer strings go to a new central control and readout unit (DAQ center) about $20 \mathrm{~m}$ below surface (see Fig. 1), where they are multiplexed to a single line to shore.

All information necessary to combine NT200 and NT+ sub-events, is provided by the common electronics module $(C E M)$ specially designed for NT200+. CEM is 
located at the DAQ center and connected through coaxial cables with the NT200 and NT+ DAQ subsystems. This module contains TDC units that measure time differences between NT200 and NT+ triggers with a resolution of about 2 ns. Also, all NT200 trigger pulses are counted and added to the NT+ data stream. Figure 7 sketches the DAQ and control system of NT200+, composed of the two subsystems for NT200 and NT+. String electronics modules (SEM) form the lower level of NT200+ DAQ system. NT200 and NT+ strings (Str) contain two and one SEM, respectively. These units translate trigger request signals from string channels to the detector electronics module $(D E M)$ or to $C E M$ for $\mathrm{NT}+$, and provide time and amplitude measurements for all triggered channels. A NT200 trigger is formed when the number of fired channels $N_{\text {hit }}$ is at least $N_{\min }$ within $500 \mathrm{~ns}$ at $D E M . N_{\min }$ is typically set to 3 or 4 . The trigger signal is used as a common stop for the TDCs of NT200 channels. For common operation with the external strings, the signal of the NT200 trigger is sent through $1.2 \mathrm{~km}$ coaxial cable to $C E M$. The number of NT200 triggers within an experimental run is recorded by a counter in CEM. On each external NT + string, triggers are formed as independent string-triggers, in case of at least 2 fired channels within $1000 \mathrm{~ns}$. String triggers are sent to $C E M$, where the time difference between string trigger and the trigger of NT200 is measured. This information is used to relate within an event the times of OMs in NT200 and the externals strings.

NT200 and NT+ experimental data are transfered to the shore center through two underwater PCs located in pressure glass spheres. Both underwater PC spheres are nearly identical, their content is detailed in Fig. 8: a single board PC/104 (PC104: Advantech-PCM9340), a DSL-modem (DSL-M: FlexDSL-PAM-SAN, with hub and router), a managed Ethernet switch (SwRSTP: RS2-4R, running RSTP protocols for the two-fold redundant ethernet network between the PC spheres), an Ethernet-ComServer (CSrv: WUT-58211, for PC-terminal emulation), two mediaconverters ( $M c$ : for coaxial connection to external control units) and the experiment data and slow-control modems ( $D$-Mod and $C$-Mod). The connection to shore is by a single DSL-Modem at a speed of up to $2 \mathrm{Mbit} / \mathrm{s}$. This full multiplexing of all data and control streams through a single DSL-channel reduces the number of shore wires to two. Both PC spheres are interconnected via two twisted pair ethernet cables (main and hot spare). This underwater system works stable since its first installation in 2004. Using Linux throughout the system (for underwater PCs and shore station PCs) allows for easy remote maintenance and control from home institutions.

\section{NT200+ Laser Calibration}

Large volume underwater Cherenkov detectors need calibration of the relative time-offsets between all light-sensors to a precision of a few nsecs, since event reconstruction and classification are based on the precise light arrival times. For NT200+, calibration is done with a powerful external laser light source with up to $5 * 10^{13}$ photons per pulse and nsec-pulse duration, which is located between two outer 
strings, see Fig.1. This ensures amplitudes of $\sim 100$ photoelectrons on a few PMTs on each external string and on NT200. High amplitudes minimize uncertainties due to light scattering.

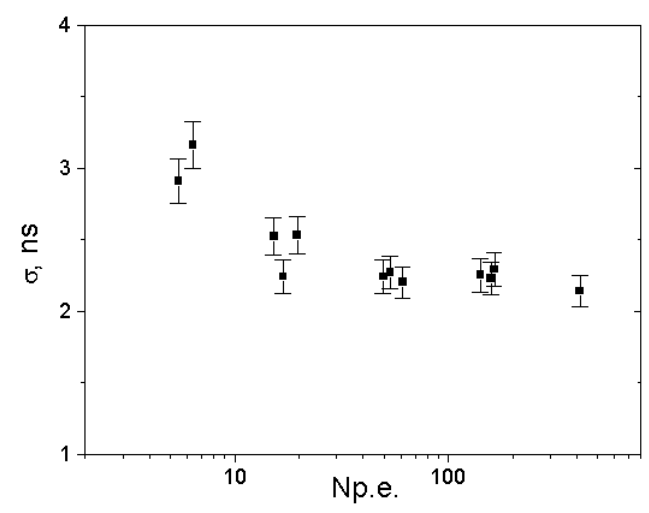

Fig. 9. Measured NT200+ time resolution as function of PMT amplitudes for laser calibration pulses.

The NT200+ laser calibration unit is made of a powerful short-pulse Nitrogen laser $(\lambda=337 \mathrm{~nm})$ with about $100 \mu \mathrm{J}$ for $<1 \mathrm{nsec}$ pulse duration, which is pumping a Coumarin dye laser at $480 \mathrm{~nm}$. After passing through a computer-controlled attenuator disc, the light is isotropized by a light diffuser ball, made of a round-bottom flask filled with Silicone Gel (RTV-6156) admixed with hollow micro-glass spheres at about $5 \%$-volume ratio (S32 from $3 \mathrm{M}$, with $\approx 40 \mu \mathrm{m}$ diameter; following an idea developed for SNO [21]). The total loss of this isotropizing sphere is $<25 \%$. All components are mounted into a $1 \mathrm{~m}$-long cylindrical glass pressure housing, which gives isotropic emission for more than the upper hemisphere. The unit is installed at a depth of $1290 \mathrm{~m}$ below surface and operated in autonomous mode: after power-on from shore, a series of pulses at various intensities is conducted.

The final light output ranges from approximately $10^{12}$ to $5 * 10^{13}$ photons/pulse, corresponding to shower energies from $10 \mathrm{PeV}$ to $500 \mathrm{PeV}$. The laser unit is used, varying the total intensity, to calibrate pointlike shower vertex and energy reconstruction algorithms for energies up to $500 \mathrm{PeV}$.

This laser unit allows for an independent performance check of the key elements of the NT200+ timing system. We performed the relative time synchronization of all news strings and NT200, and find the jitter of this to be less than 3 nsec. This jitter is due mainly to the significant length $(1.2 \mathrm{~km})$ of synchronisation line between NT200 and external strings. The measured dependence of the relative time jitter on PMT amplitudes is presented in Fig. 9 for several pairs of channels of NT200 and external strings. 


\section{Aynutdinov et al.}

\section{A Gigaton Volume Detector at Baikal}

MC simulations have shown that the detection volume of $\mathrm{NT} 200+$ for $\mathrm{PeV}$ cascades would vary only moderately, if NT200 as the central part of NT200+ is replaced by a single string of OMs. Figure 10 gives the detection volume for different configurations as a function of cascade energy. The standard configuration of NT200+ is marked by empty rectangles. The other configurations comprise a single string instead of NT200: a standard string of $70 \mathrm{~m}$ length and $24 \mathrm{OMs}$ (filled rectangles), a half string with $12 \mathrm{OMs}$ covering $35 \mathrm{~m}$ (dots), and a $70 \mathrm{~m}$ long string sparsely equipped with $12 \mathrm{OMs}$ (triangles). The configuration with the long 12-OM string shows an energy behavior very close to the one of NT200+.

For neutrino energies higher than $100 \mathrm{TeV}$, such a configuration could be used as a basic unit of a Gigaton Volume Detector (GVD). Rough estimations show that $0.7 \div 0.9$ Gton detection volume for neutrino induced high energy cascades may be achieved with about $1300 \mathrm{OMs}$ arranged at 91 strings. A top view of GVD as well as a sketch of one basic subarray are shown in Fig. 11. The physics capabilities of GVD at very high energies cover the typical spectrum of cubic kilometer arrays.

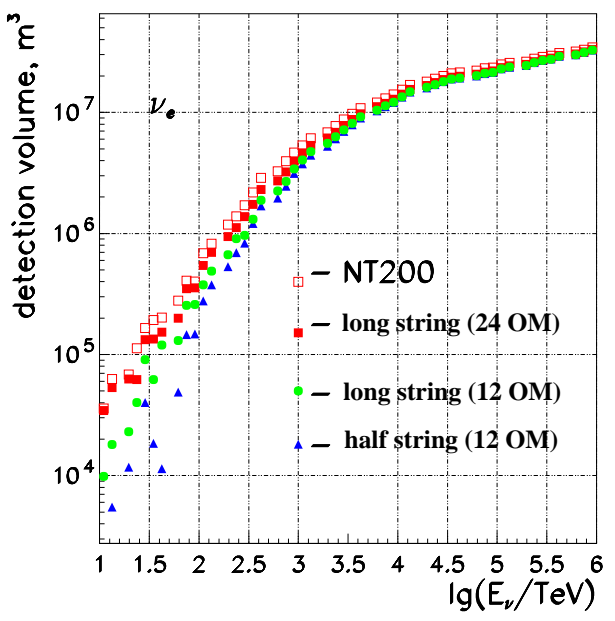

Fig. 10. Detection volumes of different configurations of the basic cell for a $\mathrm{km} 3$-size Baikal detector.

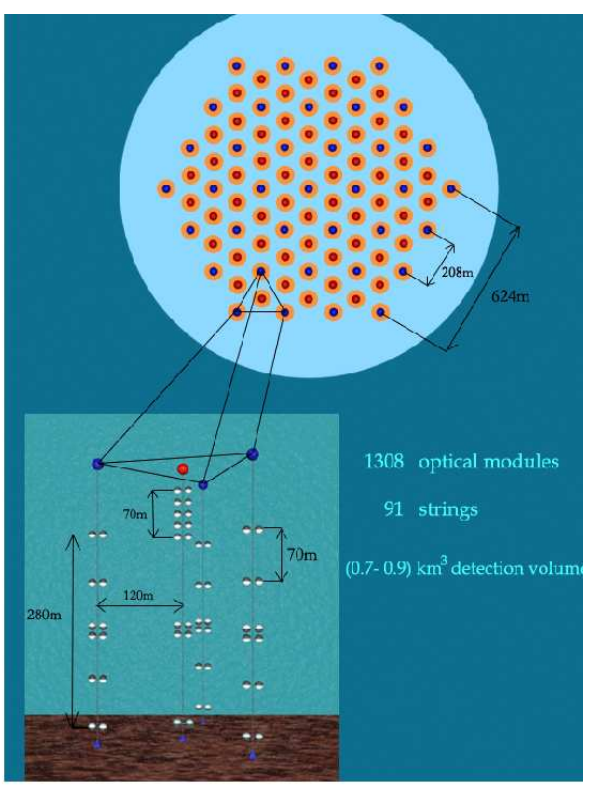

Fig. 11. Top view of the planned Baikal km3-detector (Gigaton Volume Detector). Also shown is its basic cell: a "minimized" NT200+ telescope. 


\section{Conclusions and Outlook}

The deep underwater neutrino telescope NT200 in Lake Baikal is taking data since April 1998. Using about $10^{3}$ live days, 372 neutrino induced upward going muons have been selected. Limits on the flux of diffuse astrophysical high energy neutrinos for $20 \mathrm{TeV}<\mathrm{E}<50 \mathrm{PeV}$, as well as on the $\bar{\nu}_{e}$ flux at the $W$-resonance energy have been derived. A limit on the flux of fast magnetic monopoles has been obtained.

The Baikal neutrino telescope has been significantly upgraded in 2005. The new telescope configuration NT200+ has a sensitivity better than $10^{-7} \mathrm{~cm}^{-2} \mathrm{~s}^{-1} \mathrm{sr}^{-1} \mathrm{GeV}$ for a diffuse $E^{-2}$ electron neutrino flux within the energy range $10^{2} \mathrm{TeV} \div 10^{5} \mathrm{TeV}$. NT200+ will search for neutrinos from AGNs, GRBs and other extraterrestrial sources, neutrinos from cosmic ray interactions in the Galaxy as well as high energy atmospheric muons with $\mathrm{E}_{\mu}>10 \mathrm{TeV}$. For the first 1000 hours of operation, $7.5 \cdot 10^{4}$ common events (detected by NT200 and external strings) have been collected. Data analysis is in progress.

For the planed km3-detector in lake Baikal, R\&D-activities have recently been started. With a Technical Design Report scheduled for 2008, deployment will start in 2010 .

This work was supported by the Russian Ministry of Education and Science, the German Ministry of Education and Research and the Russian Fund of Basic Research (Grants 02-452-12-7043, 05-02-17476, 04-02-17289, 04-02-16171, 05-02-16593), and by the Grant of the President of Russia NSh-1828.2003.2.

\section{References}

[1] I. Belolaptikov et al., Astropart. Phys. 7, 263 (1997).

[2] R.I. Bagduev et al., Nucl. Instrum. Methods A 420, 138 (1999).

[3] I. Belolaptikov et al., Astropart. Phys. 12, 75 (1999).

[4] I.Belolaptikov et. al. (Baikal collaboration), 26th ICRC, Salt Lake City, V.2, 340 (1999).

[5] S.Orito et. al., Phys.Rev.Lett. V.66, 1951 (1991).

[6] M.Ambrosio et. al. (MACRO collaboration), hep-ex/02007020 (2002).

[7] P.Niessen, C.Spiering for AMANDA collaboration, 27th ICRC, Hamburg, V.4, 1496 (2001).

[8] M. Ackermann et al., Astropart Phys. 22 (2004) 127.

[9] M. Ackermann et al., Astropart. Phys. 22 (2005) 339.

[10] K. Muenich et al., Proc. 29th ICRC Pune, India, 2005; Preprint astro-ph/0509330.

[11] M. Ambrosio et al., Nucl. Phys. (Proc. Suppl.) B110 (2002) 519.

[12] V. Berezinsky et al., Astrophysics of Cosmic Rays, North Holland (1990).

[13] V. Berezinsky, arxiv.gov: astro-ph/0505220 (2005). 
[14] E. Waxman and J. Bahcall, Phys. Rev. D59 (1999) 023002.

[15] K. Mannheim, R.J. Protheroe and J.P. Rachen, Phys. Rev. D63 (2001) 023003.

[16] F. Stecker and M. Salamon, astro-ph/9501064 (1995).

[17] D. Semikoz and G. Sigl, arXiv:hep-ph/0309328.

[18] L. Nellen, K. Mannheim and P. Biermann, Phys. Rev. D47 (1993) 5270.

[19] L.V. Volkova, Yad. Fiz. 31 (1980) 1510; Sov. J. Nucl. Phys. 31 (1980) 784.

[20] L.V. Volkova and G.T. Zatsepin, Phys. Lett. B462 (1999) 211.

[21] R.J. Ford, PhD Thesis, Queen's University at Kingston, Canada, 1999.

[22] V. Aynutdinov et al., arXiv:astro-ph/0508675, Astropart. Phys. 25, 140 (2006).

[23] V. Aynutdinov et al., Proc. 29th ICRC Pune, India, 2005; Preprint astro-ph/0507713.

[24] V. Aynutdinov et al., Proc. 29th ICRC Pune, India, 2005; Preprint astro-ph/0507715.

[25] V. Aynutdinov et al., Proc. 29th ICRC Pune, India, 2005; Preprint astro-ph/0507709. 